\title{
A Review of Embedding Hexagonal Cells in the Circular and Hexagonal Region of Interest
}

\author{
Marina Prvan ${ }^{1}$, Julije Ožegović ${ }^{2}$ \\ Department of Electronics and Computing \\ FESB \\ Split, Croatia
}

\author{
Arijana Burazin Mišura ${ }^{3}$ \\ Department of Mathematics and Physics \\ University Department of Professional Studies \\ Split, Croatia
}

\begin{abstract}
Hexagonal cells are applied in various fields of research. They exhibit many advantages, and one of the most important is their possibility to be closely packed and to form a hexagonal grid that fully covers the Region of Interest (ROI) without overlaps or gaps. ROI can be of various geometrical shapes, but this paper deals with the circular or hexagonal ROI approximations. The main purpose of our research is to provide a short review on the literature concerning the hexagonal grid, summarizing the existing state-of-the-art approaches on embedding hexagonal cells in the targeted ROI shapes and offering application-specific advantages. We report on formulas and algebraic expressions given in the existing researches that are used for calculating the number of embedded inner hexagonal cells or their vertices and/or edges. We contribute by integrating all researches in one place, finding a connection between previously unrelated applications concerning the use of embedded hexagonal grid and extracting commonality between previous researches on whether it provides the formulas on calculating the inner hexagon cells. In case only the number of edges or vertices is provided for the targeted application, we derive formulas for calculating the number of inner hexagons. Therefore, our survey results with the overview on solving the problem of embedding hexagonal cells in the desired circular or hexagonal ROI. The contribution of the review is the following: first it provides the existing and the derived formulas for calculating the embedded hexagons and second, it provides a theoretical background that is necessary to encourage further research. Namely, our main motivation, that is the geometrical design of the one of the world's largest CERN particle detectors, Compact Muon Solenoid (CMS) is analyzed as a source for the future research directions.
\end{abstract}

Keywords-Detector design; hexagon tessellation; region of Interest; regular grid

\section{INTRODUCTION}

Hexagonal grids are used in many different scientific fields, due to its advantages compared to other approaches. According to some researches, it has been found that using a hexagonal instead of a traditional square grid provides better results in digital image and signal processing [1-7]. In biological application, the advantage of using hexagons is present in observations, simulations and experiments, with a special benefit provided in biological modelling [8].

Many authors prompt the hexagonal grid usage, especially in cartography, because of the possibility to derive smaller resolutions by applying decomposition of the larger cells into smaller ones $[9,10]$. The use of a hexagonal cells is inevitable, since hexagonal grid offers many general advantages compared to other regular grids that use square or rectangular arrays. First, resource savings can be obtained. For example, when applied in engineering, hexagonal sensor shape enables reduced material waste when it is produced from a circular silicon wafer $[11,12]$. Also, the data processing is faster with the hexagonal structure and the needed memory usage for data storage is decreased (13.4\% compared to rectangular grid [13]). Also, the processing resources are reduced $[14,15]$.

Besides, important advantage of a hexagonal grid is that the nearest cells are equidistant from a single rounded cell, such that the nearest neighbor finder algorithm is less ambiguous than with using squares or rectangles [8, 15, 16]. Another advantage of the hexagonal grid is the possibility to fully cover the Region of Interest (ROI) without void or gaps. The increased area usage is shown with hexagons compared to other polygon shapes $[17,18]$.

There are many applications of the general hexagon usage, but not so many address a specific problem of embedding the hexagonal cells in a selected ROI. Also, according to our knowledge, no paper based on the general survey on the former question is found in the existing literature. Therefore, the aim of this paper is to provide a short review on the current topic. We concentrate on a specific case where ROI is approximated with a circular or a hexagonal shape. We classify the papers from the literature in two main classes depending on the ROI used. Next, subclasses are derived based on the practical application or the certain field of research. Further and final categorization is done based on the mathematical formulas that are provided in the paper, whether for the calculation of the total number of hexagonal cells embedded in the ROI, or the number of the corresponding inner hexagon vertices and edges.

In case that the number of inner hexagonal cells is not provided in the referent papers, we derive formulas for the total number of hexagons embedded in the ROI. The goal of this overview is to provide the existing mechanisms of hexagon cells calculation in the hexagonal and circular ROI, with the aim to develop a framework for sensor modelling in the sensing region. Our targeted application is the Compact Muon Solenoid (CMS) detector geometry, which should be designed and analyzed. The basic intention is to calculate the total number of hexagonal sensors needed to cover a sensing layer in the hexagonal modular and circular detector region to enable the evaluation of the sensor production cost in the future work. 
The paper is organized as follows. In Section II, methodology for the survey is given with the posed research questions and context. Also, the search and selection result of the literature papers is summarized in tables based on the predefined categorization strategy. Section III deals with the theoretical background on the hexagonal grid and the ROI definition. A short review on the hexagonal cells embedded in the circular and hexagonal ROI is given in Section IV. The research findings are summarized in Section V. Definition of the new CMS detector geometry model is presented in Section VI and the future research directions are derived. Section VII concludes the paper followed by references used.

\section{Methodology}

\section{A. Research Questions}

With this survey, we intend to answer the following research questions:

- RQ1: Which applications can be found in the literature that deal with embedding the hexagonal cells in circular or hexagonal ROI?

- RQ2: Are there algebraic expressions provided in the literature that calculate the number of the ROIembedded hexagonal cells and/or the number of their vertices and edges?

- RQ3: How to derive formulas which provide calculation of the embedded inner hexagonal cells in the ROI?

\section{B. Research Context}

The search strategy was applied to find the relevant papers published in conferences and journals. Hence, a manual and automatic search was done by using the IEEE Xplore and Google Scholar. A search concatenated string used as a basic criterion for the paper collection was: "hexagon", "region of interest", "packing" and "circle". Only studies published in English were included in the review and rejected otherwise.

When the initial set of papers is obtained, we excluded the duplicates. Each papers' title, abstract and keywords were analyzed to evaluate its relevance. Papers were kept if they addressed the RQs and excluded otherwise. The total collection of papers divided into conferences ( 5 papers) and journals (37 papers) is given in Table I.

\section{Paper Search and Selection Result}

Papers were divided in two basic classes depending on whether a circular or a hexagonal ROI is used. Next, the papers from each class are separated based on the specific application. The search and selection results are given in Table II for circular and Table III for hexagonal ROI shape.

TABLE I. PAPERS DIVIDED BY TYPE

\begin{tabular}{|l|l|l|}
\hline \multirow{2}{*}{ Paper type } & Extracted papers & Total \#papers \\
\cline { 2 - 3 } & References & 5 \\
\hline Conferences & {$[1],[4],[7],[16],[32]$} & 37 \\
\hline Journals & {$[2],[3],[5],[6],[8-15],[17-31],[33-42]$} & \\
\hline Total \#papers & 42 \\
\hline
\end{tabular}

TABLE II. PAPERS USING CIRCULAR ROI (CLASS 1)

\begin{tabular}{|l|l|l|l|}
\hline \multirow{2}{*}{ Application } & \multicolumn{3}{|l|}{ Provided Mathematical Formulas } \\
\cline { 2 - 4 } & \#hexagonal cells & \#vertices & \#edges \\
\hline Sensor manufacturing & {$[12]$} & {[]} & {[]} \\
\hline WSN & {$[21]$} & {[]} & {[]} \\
\hline Total \#papers & 2 & \multicolumn{3}{|l}{} \\
\hline
\end{tabular}

TABLE III. PAPERS USING CIRCULAR ROI (Class 2)

\begin{tabular}{|l|l|l|l|}
\hline \multirow{2}{*}{ Application } & \multicolumn{3}{|l|}{ Provided Mathematical Formulas } \\
\cline { 2 - 4 } & \#hexagonal cells & \#vertices & \#edges \\
\hline $\begin{array}{l}\text { Interconnection } \\
\text { networks }\end{array}$ & {$[22],[24]$} & {$[22-24]$} & {$[22],[23]$} \\
\hline $\begin{array}{l}\text { Discrete applied } \\
\text { mathematics }\end{array}$ & {$[25]$} & {$[25-28]$} & {$[25],[26]$} \\
\hline Silicate Networks & {[]} & {$[29],[30]$} & {$[29],[30]$} \\
\hline $\begin{array}{l}\text { Wireless Sensor } \\
\text { Networks (WSN) }\end{array}$ & {$[32],[34-36]$} & {$[32]$} & {[]} \\
\hline Hierarchical grids & {$[8],[9],[37],[40]$} & {[]} & {[]} \\
\hline Total \#papers & 17 & \multicolumn{2}{|l}{} \\
\hline
\end{tabular}

We further categorized the papers from each application in sub-classes depending on whether authors provide formulas on how to calculate the total number of embedded hexagonal cells, their vertices or edges.

\section{THEORETICAL BACKGROUND}

\section{A. Hexagonal Grid}

Hexagonal grid is formed by a regular tessellation of hexagonal cells used to cover the ROI without overlaps or gaps ([19]).

The hexagonal coordinate system can be defined by using various definitions [20], and we present the basic one with using two coordinate axes at $\pi 3$ angle so that a discrete indexation of each hexagonal cell becomes possible, as shown in Fig. 1.

\section{B. ROI Definition}

ROI can be approximated by any geometrical shape, but in this paper, we are interested in applications where ROI is a circle or a hexagon.

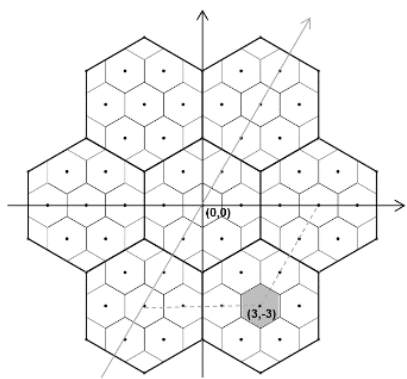

Fig. 1. An Array of Cells in the Hexagonal Grid. 


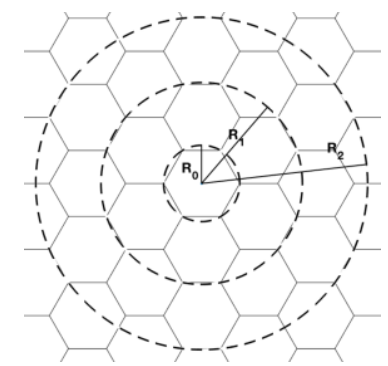

Fig. 2. Hexagonal Grid Embedded in the Centered Circle.

Based on the conducted survey, there are more papers dealing with hexagonal ROI application, even though ROI approximation with a circle is more appropriate because of the simplicity of the mathematical calculations. The process of embedding hexagonal grid into circle can be equaled by the intent to encircle the hexagonal cells in the grid, where the circle center is shared with the central hexagonal cell in the coordinate system origin $(0,0)$. However, there are many possible ways of grid encircling, as given on Fig. 2.

Also, ROI can be approximated by some other geometrical shape such as a polygon rather than circle, and we concentrate on the applications where ROI is a hexagon. The selection of these two ROI shapes is application-driven, as we target a potential geometry model for a specific application such as CMS detector sensing layer design. Our goal is to explore the state-of-the-art mathematical formulas provided in the literature or derived from the findings. Hence, in this paper, a short comprehensive review was conducted to describe, compare, and analyze the existing approaches or a related work on embedding hexagons into hexagonal or circular ROI. These are discussed thoroughly in the next section.

\section{REVIEW ON HEXAGONS EMBEDDED IN THE ROI}

\section{A. ROI Approximated by a Circle}

In sensor manufacturing, circular ROI is a silicon wafer from which hexagonal sensors are fabricated. In WSN, network sensing field or covered range can be depicted by a circle of a given radius, which is a ROI in the current context.

Embedding hexagonal grid in the circular geometrical shape is interesting to study because of the many ways it can be encircled. The number of hexagonal cells embedded in the ROI must be calculated and adjusted accordingly, so that they are entirely contained inside a circle, or some compromises must be agreed on the ROI border.

1) Sensor manufacturing: Bothra [11] considered forming semiconductor dies from smaller hexagonal sensor cells embedded on the circular wafer. Author used a hexagonal grid embedded into a circular ROI, to show that larger area can be used on wafer with using hexagons compared to other geometrical shapes. The waste of the silicone material thrown away is reduced as well as the total production cost.

There is an attempt from Davis and Sinha [12] on forming the central hexagonal polygon dies, and several smaller outer polygons arranged around the central die (Fig. 3). Authors show that hexagonal grid enables more polygon sensors to be produced compared to a square grid when the wafer radius is constant. Authors provide examples on calculating the total number of embedded sensor cells in Table IV but do not provide formulas for their calculation.

We reconstruct the total inner hexagonal or squared cells number according to Table IV as:

$$
\begin{aligned}
& N_{\text {hexagons }}(k)=3 k^{2}+3 k+1 \\
& N_{\text {squares }}(k)=4 k^{2}
\end{aligned}
$$

In both cases, parameter $\mathrm{k}$ is number of "rings", or maximum number of dies from the central die to the border (if there is central die in the tessellation like in hexagonal case, counting starts from 0 , otherwise it starts from 1). If the full tessellation does not place all the full dies on the wafer, some dies in the most outer ring are cut out by the wafer radius. Those dies are taken out from the total number of embedded sensor cells given by the formulas.

2) WSN: Unlike other grid-based regular deployment patterns in WSN, it is shown that hexagonal grid provides better coverage efficiency since it can cover the sensing area better than triangles and squares [17]. Kim et al [21] use a hexagonal grid with an ideal cell size to deploy the underwater WSN. Authors show that for a circular ROI, one can calculate number of hexagon rings needed to fully cover the disk of a given radius (Fig. 4).

TABLE IV. CALCUlating \#SENSOR Cells [12]

\begin{tabular}{|l|l|l|l|}
\hline $\begin{array}{l}\text { Side length } \\
\mathbf{a}[\mathbf{m m}]\end{array}$ & $\begin{array}{l}\text { Area per die } \\
{\left[\mathbf{m m}^{\mathbf{2}}\right]}\end{array}$ & \# square dies & $\begin{array}{l}\text { \# equivalent } \\
\text { hexagon dies }\end{array}$ \\
\hline 10 & 100 & 594 & 649 \\
\hline 20 & 400 & 148 & 151 \\
\hline 30 & 900 & 52 & 61 \\
\hline
\end{tabular}
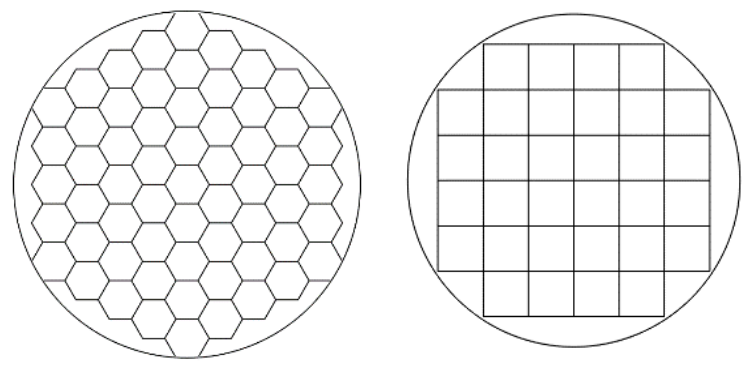

Fig. 3. Calculating \#Sensor Cells (Adjusted from [12]).

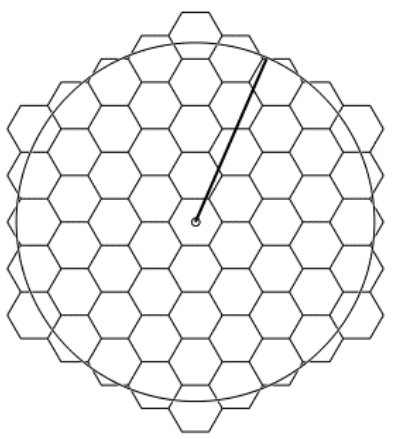

Fig. 4. Hexagon Ring Calculation in WSN (Adjusted from [21]). 
The applied formula to calculate the number of embedded hexagonal cells is the following [21]:

$N_{\text {hexagons }}= \begin{cases}\frac{(3 q+1) l}{2}=R & \text { if } q \text { is odd } \\ \frac{l \sqrt{(3 q+1)^{2}+3}}{2}=R & \text { if } q \text { is even }\end{cases}$

Therefore, the total number of hexagon rings is denoted by $\mathrm{q}$, and the small hexagon side length is given by 1 . To calculate q needed to fully cover the ROI of radius R, relation (2) must be satisfied. The number of hexagons in each hexagon ring $k$ is given by the following formula [21]:

$N_{\text {hexagons }}=6 k, k=1,2 \ldots$

Equation (3) enables one to calculate the number of hexagon rings. For example, having ROI of radius $R=100$ and given the cell size $l=9.991$, the number of hexagon rings $q=7$. Thus, we can calculate the number of hexagonal cells need to be fit inside a pre-defined circular ROI.

\section{B. ROI Approximated by a Hexagon}

1) Interconnection networks: Interconnection network consists of nodes processing the data in parallel. It is often modelled by the honeycomb network (HN) or the hexagonal network (HX) naturally contained into a hexagonal ROI. The various topology characteristics are studied depending on the application. For example, authors derive formulas to calculate the number of smaller hexagons inside the larger one, as well as the number of inner small hexagon edges or vertices.

The basis is the work of Stojmenovic [22], presenting the problem of building a hexagonal grid or how to construct a honeycomb network. Example of $\mathrm{HN}(\mathrm{t})$, where the radius $\mathrm{t}$ is the number of hexagon rings between the network center and the ROI border is shown on Fig. 5. We can see how a honeycomb hexagonal mesh network is contained or embedded inside a regular hexagonal ROI.

The total number of vertices of $\mathrm{HN}(\mathrm{t})$, is calculated as $6 \mathrm{t}^{2}$ and the total number of edges is $9 t^{2}-3 t$. The diameter (the largest distance between two nodes) is $d=4 t-1$. These parameters are important for evaluating the network performance and cost influenced by the total number of network parallel nodes and links.

We derive the size of the $\mathrm{HN}(\mathrm{t})$ in terms of total number of hexagons:

$N_{\text {hexagons }}=3 t^{2}-3 t+1$

Chen et al. [23] proposed how to count the number of nodes $\mathrm{n}$ as a function of a given distance $\mathrm{t}$ from the center node to the network vertex in a hexagonal network $\mathrm{HX}(\mathrm{t})$ presented on Fig. 6. The total number of nodes of $\operatorname{HX}(t)$ is $n=3 t^{2}-$ $3 t+1$, the total number of edges is defined as e $=9 t^{2}-15 t+$ 6 . The diameter (number of links on the hexagonal ROI circumscribed circle) is $\mathrm{d}=2 \mathrm{t}-2$.

Authors did not provide formulas for calculating the total number of embedded hexagonal cells. Therefore, we derive the formula based on the total edges/vertices count:
$N_{\text {hexagons }}(t)=3 t^{2}-9 t+7$

All inner nodes in the network can represent the center of one hexagonal cell, so if overlapping is allowed, the number of hexagons is provided by (5a). Number of hexagons in nonoverlapping case can be calculated with the following formula, where $\mathrm{t}=2 k+l, k \in N_{0}, l \in\{0,1\}$ :

$N_{\text {hexagons }}= \begin{cases}3 k^{2}-3 k+1 & \text { if } l=0 \\ 3 k^{2}-k & \text { if } l=1\end{cases}$

All parameters $\mathrm{n}, \mathrm{e}, \mathrm{d}$ and the number of hexagons is considered as a function of $t$, representing the size of the network [24]. Honeycomb network $\mathrm{HC}(\mathrm{n})$ is obtained from $\mathrm{HC}(\mathrm{n}-1)$ by adding a ring of hexagons around the boundary of $\mathrm{HC}(\mathrm{n}-1)$ [25]. It means that the number of nodes in each hexagon ring up to $t$ equals (3), and the total number is again a cumulative sum (4).

2) Discrete applied mathematics: We can consider a problem of defining hexagonally shaped honeycomb and hexagonal networks to be the same as embedding hexagonal cells in a hexagonal ROI. This is a well-known problem in applied discrete mathematics, where a connection between hexagonal and honeycomb networks is studied. By the definition, every honeycomb network $\mathrm{HN}(\mathrm{t}-1)$ is contained inside some hexagon network $\operatorname{HX}(t)[25,26]$. Considering the example on Fig. 7, it is clearly indicated that, to embed a hexagonal grid in the hexagonal ROI, one needs to find its dual HX. Also, in the opposite procedure, the dual HN embedded in the HX of the ROI can be examined. The number of hexagons is again (4).

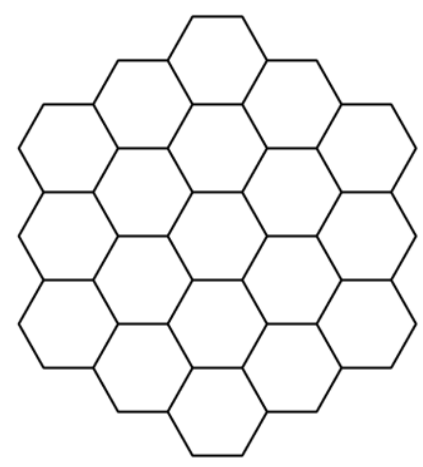

Fig. 5. Honeycomb Network HN (3) (Adjusted from [22]).

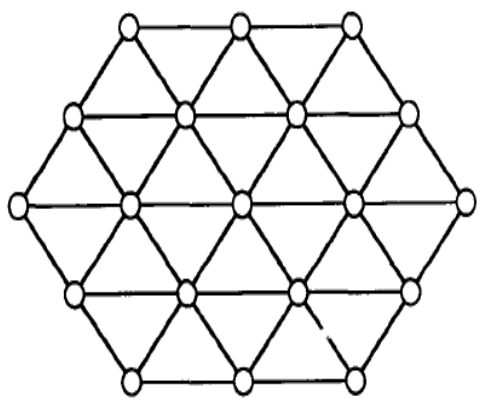

Fig. 6. Hexagonal Network HX (3) [23]. 


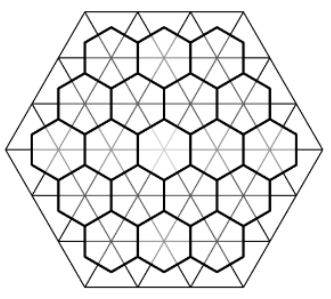

Fig. 7. HN (3) is Contained Inside HX (4) (Adjusted from [26]).

A mathematical problem of embedding a hexagonal grid into a bounding hexagon is presented in the literature as a degree diameter problem considering a graph as being embedded in host graph such as honeycomb network. This problem involves finding the largest sub graph in terms of the number of vertices subject to constraints on the degree and the diameter [27]. Diameter D is total number of edges on the circumscribed circle diameter of the hexagon by which the graph is encircled (Fig. 8).

In the former context, a host graph is encircled by a hexagonal ROI with the size given by D. Formulas are developed to find the total number of inner vertices, divided into classes based on the odd and even diameter size [27]:

$\left|V\left(H_{D}^{2}\right)\right|= \begin{cases}6 k^{2}+3 k+1, & \text { when } r=0 \\ 6 k^{2}+6 k+2, & \text { when } r=1 \\ 6 k^{2}+9 k+4 & \text { when } r=2 \\ 6 k^{2}+12 k+6 & \text { when } r=3\end{cases}$

In the above formulas, $\mathrm{H}$ is the honeycomb grid, where $H_{D}^{2}$ denotes a largest subgraph of $\mathrm{H}$ of diameter $\mathrm{D}=4 k+$ $r, k, r \in N, r \in\{0,1,2,3\}$. Depending on the ROI size, for it to be a hexagonal shape, the diameter must be even. Also, for $D=4 k$, ROI is a regular hexagon and for $D=4 k+2$, ROI is an irregular hexagon. Fig. 8 gives an example for both. Authors in [27] do not provide the total number of hexagons embedded inside the ROI. We derive the former with the following formulas:

$N_{\text {hexagons }}=\left\{\begin{array}{c}3 k^{2}, \quad \text { when } r=0 \\ k(3 k+3), \text { when } r=2\end{array}\right.$

There is a similar attempt to solve the degree diameter problem while counting the total number of vertices of the subgraph based on the triangular grid which is the basis for hexagonal networks [28]. It is expressed as a function of diameter, defining two classes of architectures, depending on weather the diameter is even or odd:

$N_{T}(D)= \begin{cases}\frac{3}{4} D^{2}+\frac{3}{2} D+\frac{3}{4} & \text { for odd } D \\ \frac{3}{4} D^{2}+\frac{3}{2} D+1 & \text { for even } D\end{cases}$

Again, even diameter size architecture explicitly touches upon an issue of embedding hexagonal grid into a hexagon. Also, even diameter size is implying a regular hexagonal ROI, while the odd diameter size is used when ROI is the irregular hexagon (Fig. 9). Equation (8) enables one to calculate the total number of inner cells' vertices [28]. We derive formulas to calculate the total number of inner hexagons:
$N_{\text {hexagons }}=\left\{\begin{array}{l}\frac{3}{4}(D-2)^{2}+\frac{3}{2}(D-2)+\frac{3}{4} \text { for odd } D \\ \frac{3}{4}(D-2)^{2}+\frac{3}{2}(D-2)+1 \text { for even } D\end{array}\right.$

Formula (9a) allows overlapping hexagons. If overlapping is not allowed, and $\mathrm{D}=4 k+r, k, r \in N$ the total number of hexagons is given by:

$N_{\text {hexagons }}= \begin{cases}3 k^{2}-k & \text { when } r=0 \\ 3 k^{2}+k & \text { when } r=1 \\ 3 k^{2}+3 k+1 & \text { when } r=2 \\ 3 k^{2}+3 k+1 & \text { when } r=3\end{cases}$

3) Silicate networks: There are researches in which embedding of the honeycomb and hexagonal networks into silicates are studied. Naturally, a hexagonal ROI shape is used. For example, Manuel et al. [29] show a silicate network can be constructed from hexagons. Authors calculate the total number of hexagon nodes and edges in the network. HX (n) node labeling before a silicate network construction is given on Fig. 10.

One can see on the figure that hexagons are clearly emphasized inside a hexagonal ROI. However, authors do not provide their total number. We derive the former with the following expression:

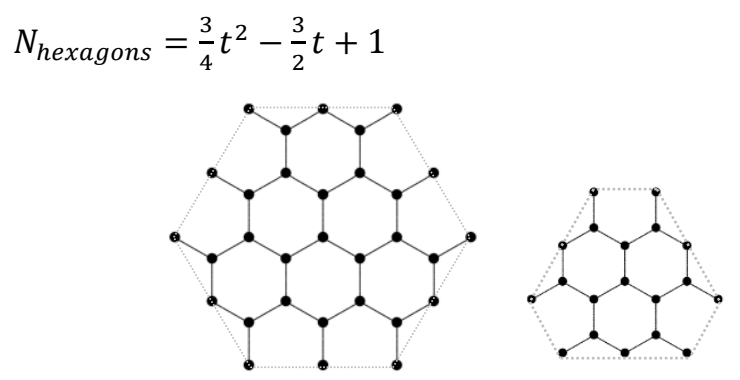

Fig. 8. Honeycomb Grid with $\mathrm{D}=8$ and $\mathrm{D}=6$ (Adjusted from [27]). We Added the Light Gray Border to Visualize the ROI Shape.

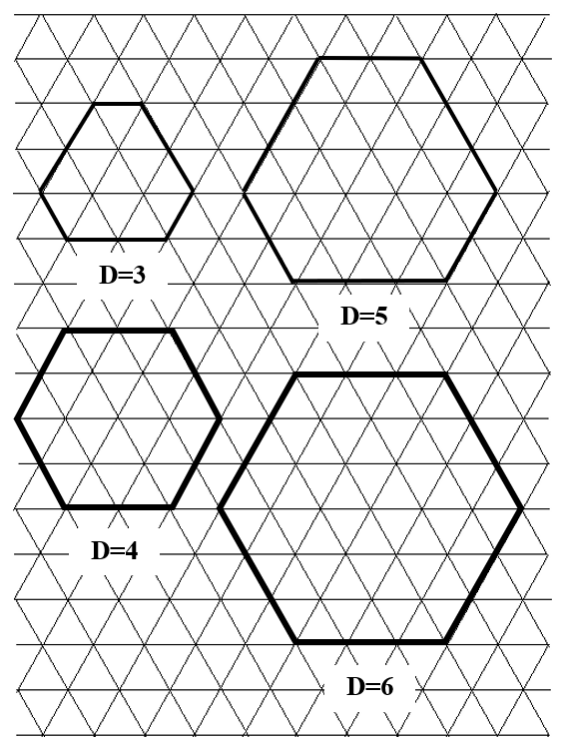

Fig. 9. The Graphs for $\mathrm{D}=3,4,5,6$ (Adjusted from [28]). 


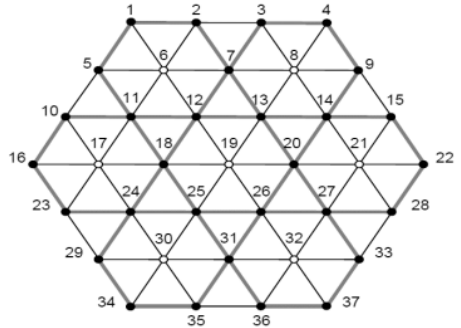

Fig. 10. HX Node Labeling [29]. White Nodes Represent Centers and Black Nodes are Vertices of the Inner Hexagonal Cells.

Parameter $t$ in the upper formula (10) is a total number of vertices from the central node to the network vertex.

In the former context, there are some other papers addressing the construction of the new, not only hexagon-like structures, derived from hexagonal and honeycomb networks with using graph operations. Basic properties of the defined topologies are investigated, such as the total number of hexagon vertices and edges [30].

4) WSN: To enable an efficient area coverage in WSN, it is not enough to position sensors only at vertices of the hexagon cells in a hexagonal grid. When covering a large hexagonal or circular area with WSN, sensors should also be placed at the centers of hexagon cells to eliminate voids.

The total number of hexagons can be calculated with a known formula for centered hexagonal numbers (4) [31]. Parameter $\mathrm{n}$ in formula (4) can be perceived as $n^{\text {th }}$ ring of hexagons [32]. As given on Fig. 11, authors place sensors on hexagon vertices and centers to form a hexagonal WSN grid.

Also, a total number of deployed sensors or nodes marked in Fig. 11, is calculated by the equation below [32]:

$N_{\text {sensors }}=a+\sum_{i=0}^{N}(b+c(N-i) d)$

In (11), $\mathrm{N}$ is number of hexagon rings required for the coverage, $a=7$ as equal to the innermost ring where total number of sensors is $7, \mathrm{~b}$ constant in the relation adds 6 hexagons with 4 nodes per hexagon i.e., total number of nodes is 24. Constant $c=6$, because for each ring, the multiple of 6 hexagons is added. Constant $d=3$, because for each added hexagon, 3 additional nodes are added.

Another example of embedding hexagonal cells into a hexagonal ROI can be found in WSN clustering example. It is shown that this structure provides better connectivity, where the sensing nodes are deployed at the vertices and centers of each hexagon cell [33]. Fan has shown that calculating not only number of cells in the ROI, but also the cell area in a hexagonal cluster group is interesting [34]. The total number of hexagonal clusters is $6 i^{2}$ and the number of hexagonal clusters in each ring is defined with the following formula, where $\mathrm{i}$ is the $i^{\text {th }}$ ring from the center $[34,35]$ :

$C_{i}= \begin{cases}6, & \text { if } i=1 \\ 6(i-1), & \text { if } i \geq 2\end{cases}$

There are a lot of benefits for WSN by using hexagonal clusters [36]. The idea of the subdivision, illustrated in Fig. 12 (a), is to divide a hexagonal ROI into a set of smaller sub- hexagon cells, for example a subdivision $R / 3$ into a group of 7 sub-hexagons and 6 third sub-hexagons, which is in total 9 hexagons. Deeper subdivision example is shown on Fig. 12 (b), a subdivision R/4 into 13 sub-hexagons and 6 half subhexagons. Authors show that for subdivision $\mathrm{R} / \mathrm{n}$ total number of equal hexagonal cells is $n^{2}$.

5) Hierarchical hexagonal grids: The use of hierarchical grids is common in cartography and biological modelling, where the basic intention is to simplify a grid by reducing its resolution [8]. There are several papers trying to modify the resolution of a grid by decomposing larger cells into smaller ones. Authors describe scaling schemes in which the use of partial hexagons is of interest.

Sahr et al. [37] have shown earlier that a large hexagon cannot be composed of entire smaller hexagons. Hexagonal grids need to have a combination of hexagonal and nonhexagonal composition. As visualized on Fig. 13, one uses 4 hexagons ( 1 full and 6 border hexagon halves) and the other has 9 hexagons ( 7 full hexagons and 6 border hexagon thirds).

Ben et al. [38] derived the possibility of ROI rotation. They develop two specific definition schemes for embedding hexagonal grid in the hexagonal ROI (Fig. 14). The approach for the inner scheme is presented also by Kumar et al. [39]. While both former papers provide a model for embedding hexagons into hexagons, no formulas are derived for the total number of inner hexagon cells.

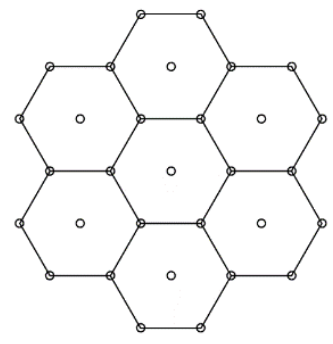

Fig. 11. WSN using Hexagonal Grid Deployment.
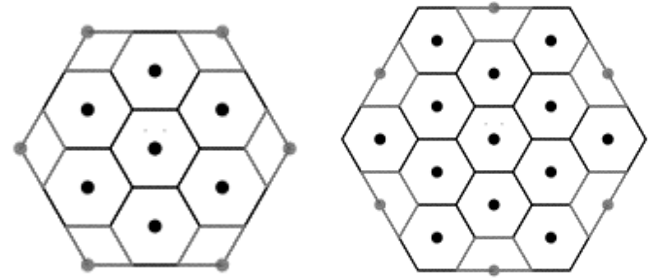

Fig. 12. WSN Cluster Subdivision (a) R/3 (b) R/4 (Adjusted from [36])

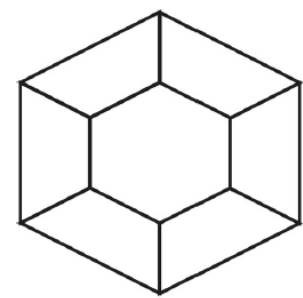

(a)

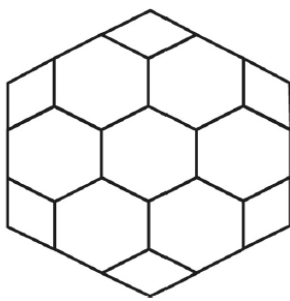

(b)
Fig. 13. Decomposition of Hexagonal ROI in: (a) 4; (b) 9 Hexagons [8, 37]. 


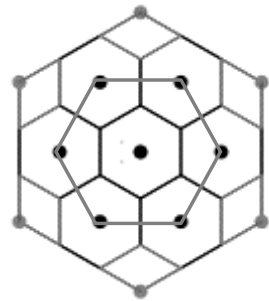

Fig. 14. Embedding Hexagons Into a Hexagonal ROI (Adjusted from [38]).

Sahr et al. [9] calculate the ratios between cell areas at a given grid resolution and the next larger scale grid resolution. They show that $\mathrm{N}$ hexagon cells at each finer resolution of a grid have $1 / \mathrm{N}$ the area of the next larger grid.

Examples of 1/3, 1/4 and 1/7 fraction are given in Fig. 15. These examples are referred to as centered, meaning that small cells share their centers with larger resolution cells. Vertexsharing variants are given by [40]. An example of the 1/4 fraction is presented on Figure 15 (d), whereas the vertices are shared among the small and large hexagonal cells.

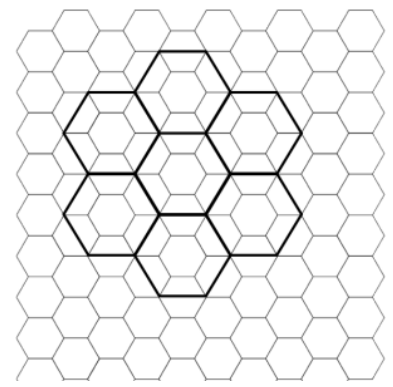

(a)

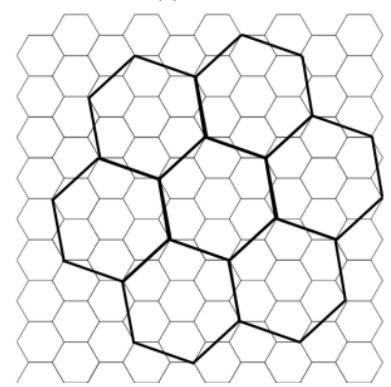

(c)

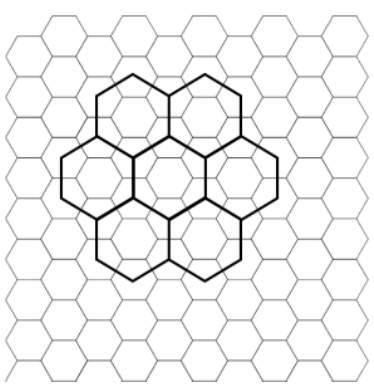

(b)

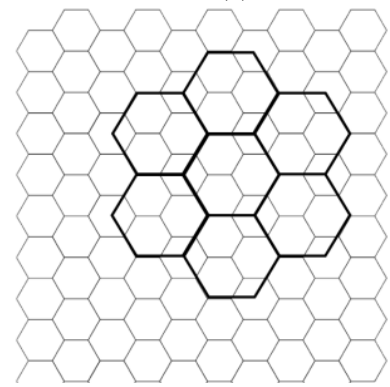

(d)
Fig. 15. Hexagonal Resolutions. Center Sharing (a) Fraction 1/3 (b) Fraction 1/4 (c) Fraction 1/7 (d) Vertex Sharing Fraction 1/4 ([9], [40]).

\section{Evaluation}

The short overview presented in the previous section provides a state-of-the-art on embedding hexagonal cells in the targeted ROI. The literature study revealed the following:

- Each specific application can consider the number of hexagon cells, hexagon vertices and/or centers. In case only vertices or edges are calculated, one can derive the total number of inner hexagons.

- Each application can consider the embedded hexagons to be overlapping or non-overlapping, regarding their total number.
- Inner hexagons can be full or partial ones, especially at the border, and their total number can be calculated separately.

- In some applications, the compromises are considered on the ROI outer border for the cells to be completely contained inside the circular or hexagonal region.

\section{A. Summarized Review Findings}

The summarized researches are complemented based on their basic purpose when hexagons are embedded in the ROI. Hence, authors in the papers are:

- Providing models on how to design a specific structural scheme that we are interested in for the CMS geometry application, but with no derived algebraic expressions for calculating the inner cells' total number ([38, 39]).

- Providing formulas for the inner hexagonal cells' number calculation inside a larger hexagon, but:

○ using a well-known formula (1) that is usually applied for the honeycomb network design [22, $24,25,34,35]$

○ only providing examples on how to calculate the total number of inner hexagons including the hexagonal parts such as halves of thirds but with no generalized approach $[8,9,36,40]$

- Calculating the total number of inner hexagons' vertices and/or edges, and the total hexagon number can be derived based on the provided expressions [23, 27-29]

\section{FUTURE RESEARCH DIRECTIONS}

\section{A. CMS Detector Design Upgrade}

The previously summarized review findings can be used as guidelines for the further studies. The source of research is CMS detector, which is a famous general-purpose instrument for studying proton-to-proton collisions in the Large Hadron Collider (LHC) experiment at CERN [41].

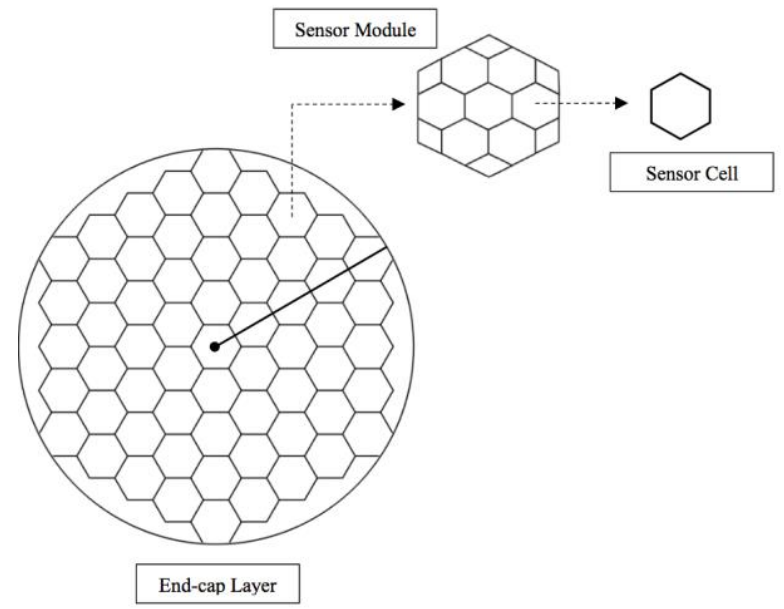

Fig. 16. Hexagonal Structure in the CMS ECAL Upgrade. 
Detector is continuously being improved, basically for two main reasons: development of new technologies and replacing parts of the detector which are damaged by very high levels of radiation. In the second phase of the LHC physics program, the upgrades are foreseen by the CMS technical proposal [42]. The most important part of the CMS detector being replaced is electromagnetic calorimeter (ECAL) end-caps since its material gets most damaged by the high levels of radiation. The new sampling ECAL design is proposed, being circular in shape and several layers deep [42]. Each layer should be covered in hexagonal sensor modules (SM) that consist of the inner hexagonal sensor cells (SC) and cover the detector circular end-cap layer (Fig. 16).

\section{B. CMS as a Source for Future Research}

As a main future research direction, we are interested in the geometry studies with the aim of the SM design for CMS. The result of the provided literature review enables the overview of the existing models for embedding hexagonal SCs into a hexagonal SM for CMS, which is a hexagonal ROI in the current context. Also, as hexagonal modules are embedded on the circular detector layer, the existing ways of embedding hexagons into a circular shape is interesting. Basic goal of the future study is the calculation of the total number of the innerROI hexagonal cells whether circular or hexagonal, to evaluate the embedding efficiency.

\section{DISCUSSION}

In Table V, the comparison between papers in the review is given based on the targeted future research. We discuss the potential of applying the state-of-the-art calculations in our targeted application-specific context.

We define several criteria for comparison, such as:

- the total number of hexagonal SCs in the hexagonal SM,

- the total number of hexagonal SMs in the circular detector layer,

- the provided geometrical model for embedding hexagonal grid in the ROI,

- the identification of the partial SCs at the ROI border.

As given in Table $\mathrm{V}$, all selected references provide a possible geometry model for the design of the CMS detector sensing layer. However, not all of them provide the possible formulas for the number of SMs inside a circular sensing region, or the number of SCs in the hexagonal ROI. Even though some basic formulas are derived from the literature findings, there are only few papers in the evaluation part of our conducted review with the potential of having the border SC types identified. Certainly, there is a clear indication for further research on detector sensing layer geometry, in contrast to the summarized review findings. Namely, the total number of inner SCs should be calculated with the objective to estimate the overall SM production cost.

\section{A. Limitations of the Study}

Some limitations of the conducted literature survey should be noted. This is a rather short review where we concentrate only on typical applications found in the literature where hexagons need to be embedded in the ROI. There may be other approaches with the similar objective, but we omit it from the review if it does not trigger the potential geometry model for our targeted application. Also, every class of applications in the review could be classified based on various criteria that are application-specific. However, we classify papers only based on the provided formulas used to calculate the number of inner hexagon cells, their edges and vertices. The reason is again the general purpose of the review, to examine the existing formulas on calculating the number of embedded hexagons inside circular or hexagonal area. Also, hexagons can be embedded in other geometrical shapes, while again, the selected ROI shapes are application driven.

TABLE V. COMPARISON BASED ON THE Future RESEARCH

\begin{tabular}{|c|c|c|c|c|}
\hline \multirow[b]{2}{*}{ Ref. } & \multicolumn{4}{|c|}{ CMS Geometry Application } \\
\hline & $\begin{array}{l}\text { \#SMs inside } \\
\text { detector layer }\end{array}$ & $\begin{array}{l}\text { \#SCs inside } \\
\text { SM }\end{array}$ & $\begin{array}{l}\text { Geometry } \\
\text { model }\end{array}$ & $\begin{array}{l}\text { Border SCs } \\
\text { identified } \\
\end{array}$ \\
\hline [1] & $x$ & $x$ & $\checkmark$ & $x$ \\
\hline [2] & $x$ & $x$ & $\checkmark$ & $x$ \\
\hline [3] & $x$ & $x$ & $\checkmark$ & $x$ \\
\hline [4] & $x$ & $x$ & $\checkmark$ & $x$ \\
\hline [5] & $x$ & $x$ & $\checkmark$ & $x$ \\
\hline [6] & $x$ & $x$ & $\checkmark$ & $x$ \\
\hline [7] & $x$ & $x$ & $\checkmark$ & $x$ \\
\hline [8] & $x$ & $x$ & $\checkmark$ & $x$ \\
\hline [9] & $x$ & $\checkmark$ & $\checkmark$ & $\checkmark$ \\
\hline [10] & $x$ & $x$ & $\checkmark$ & $\checkmark$ \\
\hline [11] & $\checkmark$ & $x$ & $\checkmark$ & $x$ \\
\hline [12] & $\checkmark$ & $x$ & $\checkmark$ & $x$ \\
\hline [13] & $x$ & $x$ & $\checkmark$ & $x$ \\
\hline [14] & $x$ & $x$ & $\checkmark$ & $x$ \\
\hline [15] & $x$ & $x$ & $\checkmark$ & $x$ \\
\hline [16] & $x$ & $x$ & $\checkmark$ & $x$ \\
\hline [17] & $x$ & $x$ & $\checkmark$ & $x$ \\
\hline [18] & $x$ & $x$ & $\checkmark$ & $x$ \\
\hline [19] & $x$ & $x$ & $\checkmark$ & $x$ \\
\hline [20] & $x$ & $x$ & $\checkmark$ & $x$ \\
\hline [21] & $\checkmark$ & $x$ & $\checkmark$ & $\checkmark$ \\
\hline [22] & $x$ & $\checkmark$ & $\checkmark$ & $x$ \\
\hline [23] & $x$ & $\checkmark$ & $\checkmark$ & $x$ \\
\hline [24] & $x$ & $x$ & $\checkmark$ & $x$ \\
\hline [25] & $x$ & $\checkmark$ & $\checkmark$ & $x$ \\
\hline [26] & $x$ & $\checkmark$ & $\checkmark$ & $x$ \\
\hline [27] & $x$ & $\checkmark$ & $\checkmark$ & $x$ \\
\hline [28] & $x$ & $\checkmark$ & $\checkmark$ & $x$ \\
\hline [29] & $x$ & $\checkmark$ & $\checkmark$ & $\checkmark$ \\
\hline [30] & $x$ & $x$ & $\checkmark$ & $x$ \\
\hline [31] & $x$ & $\checkmark$ & $\checkmark$ & $x$ \\
\hline [32] & $x$ & $\checkmark$ & $\checkmark$ & $x$ \\
\hline [33] & $x$ & $x$ & $\checkmark$ & $x$ \\
\hline [34] & $x$ & $\checkmark$ & $\checkmark$ & $x$ \\
\hline [35] & $x$ & $\checkmark$ & $\checkmark$ & $x$ \\
\hline [36] & $x$ & $\checkmark$ & $\checkmark$ & $\checkmark$ \\
\hline [37] & $x$ & $\checkmark$ & $\checkmark$ & $\checkmark$ \\
\hline [38] & $x$ & $x$ & $\checkmark$ & $\checkmark$ \\
\hline [39] & $x$ & $x$ & $\checkmark$ & $\checkmark$ \\
\hline [40] & $x$ & $\checkmark$ & $\checkmark$ & $\checkmark$ \\
\hline
\end{tabular}




\section{CONCLUSION}

In this paper, the contribution is threefold. First, we provide an overview on embedding hexagonal cells in the circular and hexagonal ROI, integrating papers from different fields of research. We classify the papers based on the ROI type and the specific application used. We find connections between previous work, concentrating on the need for embedding hexagons into circles or hexagons in the mentioned application-specific context. Next, we report on the existing formulas for calculating the number of inner hexagonal cells, or their vertices and/or edges. Finally, in case only the number of edges or vertices is provided for the targeted application, we contribute by deriving formulas for calculating the number of inner hexagons.

As the overall conclusion derived by our review, there are studies from various research domains dealing with how smaller hexagons can be embedded into larger shapes such as circular or hexagonal region. The importance of calculating the total number of inner hexagons is clearly emphasized in the literature, as well as the potential problems that arise on the outer ROI border in the packing process. Concluded by the conducted review, there exists a theoretical background for the future CMS detector geometry studies, where a hexagonal SM is designed containing hexagonal SCs, and SMs are tessellated on the circular detector sensing layer. Hence, some basic guidelines for the future research on the CMS geometrical structure is provided, together with the existing formulas and algebraic expressions that could be applied or derived from the literature findings. As a next step in our research, we intend to derive a framework of architectures that can be used for the hexagonal SM design, with the aim to efficiently embed the inner hexagonal SCs. The total sensor production cost will be evaluated. Possibly, the solution will be found for solving the problem of packing border cells, at the same time identifying the various sensor shapes that need to be produced.

\section{REFERENCES}

[1] Aiazzi, Bruno \& Baronti, Stefano \& Capanni, Annalisa \& Santurri, Leonardo and Vitulli, Raffaele. (2019). Advantages of Hexagonal Sampling Grids and Hexagonal Shape Detector Elements in Remote Sensing Imagers.

[2] Snehali A. Dakhore, K. B. Bijwe (2017): Review on Performance Analysis of Square Pixel and Hexagonal Pixel Structure in Image Processing, International Journal on Recent and Innovation Trends in Computing and Communication ISSN: 2321-8169 Vol: 5 Issue: 5, 2017.

[3] Barun Kumar, Pooja Gupta, Kuldip Pahwa: Square Pixels to Hexagonal Pixel Structure Representation Technique, International Journal of Signal Processing, Image Processing and Pattern Recognition Vol.7, No.4 (2014), pp.137-144.

[4] Fayas Asharindavida, Nisar Hundewale, Sultan Aljahdali: Study on Hexagonal Grid in Image Processing, 2012 International Conference on Information and Knowledge Management (ICIKM) IPCSIT vol.45.

[5] Lee Middleton, Jayanthi Sivaswamy: Hexagonal Image Processing, Springer-Verlag London Limited 2005; DOI: https://doi.org/10.1007/184628-203-9

[6] W. Wen \& S. Khatibi, "Virtual Deformable Image Sensors: Towards to a General Framework for Image Sensors with Flexible Grids and Forms". Sensors. 18. 10.3390/s18061856. 2018.

[7] R. Manthey, T. Schlosser \& D. Kowerko, "Generation of Images with Hexagonal Tessellation using Common Digital Cameras." IBS International Summerschool on Computer Science, Computer Engineering and Education Technology 2017.
[8] Colin P.D. Birch, Sander P. Oom, Jonathan A. Beecham, Rectangular and hexagonal grids used for observation, experiment and simulation in ecology, Ecological Modelling, Vol 206, Issues 3-4, 2007, Pages 347359, ISSN 0304-3800, https://doi.org/10.1016/j.ecolmodel.2007.03.041.

[9] Sahr K., Dumas M., Choudhuri N. (2015). "The PlanetRisk Discrete Global Grid System" [Online], http://www.discreteglobalgrids.org/wpcontent/uploads/sites/33/2016/10/PlanetRiskDGGS.pdf.

[10] Franz-Benjamin Mocnik (2018). "A novel identifier scheme for the ISEA Aperture 3 Hexagon Discrete Global Grid System", Cartography and GIS, DOI: $10.1080 / 15230406.2018 .1455157$.

[11] Bothra S. (1997). "Hexagonal Semiconductor Die, Semiconductor Substractes, and Methods of Forming a Semiconductor Die ", United States patent 6,030,885, Feb. 29, 2000.

[12] Davis, Taryn J., and Tuhin Sinha. "Polygon die packaging." U.S. Patent No. 9,911,716. 6 Mar. 2018.

[13] Sousa N. A. "Hexagonal Grid Image Processing Algorithms in Neural Vision and Prediction", Faculdade de Engenharia da Universidade, June 2014.

[14] Xiao Z., Baas B. (2013) A Hexagonal Processor and Interconnect Topology for Many-Core Architecture with Dense On-Chip Networks. In: Burg A., Coṣkun A., Guthaus M., Katkoori S., Reis R. (eds) VLSISoC: From Algorithms to Circuits and System-on-Chip Design. VLSI$\mathrm{SoC}$ 2012. IFIP Advances in Information and Communication Technology, vol 418. Springer, Berlin, Heidelberg.

[15] Li, Xiangguo. (2013). Storage and addressing scheme for practical hexagonal image processing. Journal of Electronic Imaging. 22. 010502. 10.1117/1.JEI.22.1.010502.

[16] Peter Yap: Grid based path finding; Advances in Artificial Intelligence 15th Conference of the Canadian Society for Computational Studies of Intelligence, AI 2002.

[17] P. Saritha Hepsibha and G. Sanjiv Rao (2013), "Comparative Analysis of Area Coverage in WSNs Using Various Grid-Based Node Deployment Schemes," International Journal of Future Computer and Communication vol. 2, no. 6, pp. 633-637, 2013.

[18] E. Donkoh \& A. Opoku, "Optimal Geometric Disks Covering using Tessellable Regular Polygons". Journal of Mathematics Research. 8. 25. 10.5539/jmr.v8n2p25. 2016.

[19] Weisstein, Eric W. "Hexagonal Grid." From MathWorld--A Wolfram Web Resource. http://mathworld.wolfram.com/HexagonalGrid.html.

[20] De Sousa LM, Leitão JP (2018). "HexASCII: A file format for cartographical hexagonal rasters". Transactions in GIS. 2018; 22:217232. https://doi.org/10.1111/tgis.12304.

[21] Kim S., Cheon H., Seo S., Song S. and Park S. (2010). "A hexagon tessellation approach for the transmission energy efficiency in underwater wireless sensor networks", Journal of Information Processing Systems Vol. 6. No.1, p. 53-66.

[22] Stojmenovic I. (1997). "Honeycomb networks: Topological properties and communication algorithms", IEEE Transactions on Parallel and Distributed Systems, vol. 8, no. 10, pp. 1036-1042.

[23] Chen M.S., Shin K.G., and Kandlur D.D. (1990). „Addressing, Routing, and Broadcasting in Hexagonal Mesh Multiprocessors “, IEEE Trans. Computers, vol. 39 , no. 1, pp. 10-18.

[24] García F., Solano J., Stojmenovic I., Stojmenovic M. (2003). „Higher dimensional hexagonal networks ", Journal of Parallel and Distributed Computing, Volume 63, Issue 11, p. 1164-1172, ISSN 0743-7315.

[25] Monica C.M. and Santhakumar S. (2016). "Partition Dimension of Honeycomb Derived Networks", International Journal of Pure and Applied Mathematics, Vol 108, No. 4, p. 809-818, ISSN:1311-8080.

[26] Manuel P., Bharati R., Rajasingh I., and Monica C.M. (2008). „On minimum metric dimension of honeycomb networks ", Journal of Discrete Algorithms, Volume 6, Issue 1, p. 20-27, ISSN 1570-8667.

[27] Holub P., Miller M., Pérez-Rosés H., and Ryan J., 2014. „Degree diameter problem on honeycomb networks ", Discrete Applied Mathematics, Volume 179, No. 31, p. 139-151, ISSN 0166-218X.

[28] Holub P. and Ryan J. (2015). ,Degree diameter problem on triangular networks ", Australian Journal of Combinatorics, Vol 63 (3), p. 333345 . 
[29] Manuel P., Rajasingh I., William A., and Kishore A. (2014). "Computational aspects of silicate networks", International Journal of Computing Algorithm, No. 3, p. 524-532.

[30] Hayat S., Malik M.A., and Imran M. (2015). "Computing Topological Indices of Honeycomb Derived Networks", Romanian Journal of Information Science and Technology 18, p. 144-165.

[31] Conway J. H. and Guy R. K. (1996). „The book of numbers “, New York: Copernicus.

[32] Hema N. and Krishna K. (2013). “Optimization of sensor deployment in WSN for precision irrigation using spatial arrangement of permanent crop", Sixth Int. Conference on Contemporary Computing (IC3), IEEE.

[33] Khoufi I., Minet P., Laouiti A. and Mahfoudh S. (2014). "Survey of Deployment Algorithms in Wireless Sensor Networks: Coverage and Connectivity Issues and Challenges", International Journal of Autonomous and Adaptive Communications Systems, p.24.

[34] Fan C.S. (2016). "HIGH: A Hexagon-based Intelligent Grouping Approach in Wireless Sensor Networks", Advances in Electrical and Computer Engineering, Vol. 16, No. 1, p. 41-46.

[35] Sharieh, Ahmad \& Qatawneh, Mohammad \& Almobaideen, Wesam. (2009). Hex-Cell: Modeling, Topological Properties and Routing Algorithm. European Journal of Scientific Research. 22. 457-468.

[36] Wang D., Liwei L and Li X. (2011). "A study of subdividing hexagonclustered WSN for power saving: Analysis and simulation", Ad Hoc Networks, Vol. 9, No.7, p.1302-1311.
[37] Sahr, K., D. White, and A. J. Kimerling (2003). "Geodesic discrete global grid systems". Cartography and Geographic Information Science 30 (2), 121-134.

[38] Ben, J., Li, Y., Zhou, C., Wang R., Ling Yu D. (2018): „Algebraic encoding scheme for aperture 3 hexagonal discrete global grid system ", Sci. China Earth Sci. (2018) 61: 215. https://doi.org/10.1007/s11430017-9111-yF.

[39] V. Kumar, V. Kumar, D.N. Sandeep, S. Yadav, R.K. Barik, R. Tripathi, S. Tiwari, Multihop Communication based Optimal Clustering in Hexagon and Voronoi Cell Structured WSNs, International Journal of Electronics and Communications (2018), doi: https://doi.org/10.1016/j.aeue.2018.05.025.

[40] Mahdavi-Amiri, A., E. Harrison, and F. Samavati. 2014. "Hexagonal Connectivity Maps for Digital Earth." International Journal of Digital Earth: 1-20. doi:10.1080/17538947.2014.927597.

[41] CMS Collaboration (2008), "The CMS experiment at the CERN LHC", Journal of Instrumentation, S08004. DOI: 10.1088/17480221/3/08/S08004.

[42] CMS Collaboration (2015). "Technical Proposal for the Phase-II Upgrade of the Compact Muon Solenoid", CERN-LHCC-2015-10, ISBN 978-92-9083-417-5. 